Durch die Spezialisierung und die Fortschritte in der Behandlung sind immer mehr Fachleute in die Abklärungs- und Behandlungskette eingebunden. Patientinnen und Patienten werden deshalb während einer Krankheitsphase sequenziell oder parallel von verschiedenen medizinischen und anderen Fachpersonen behandelt. Entsprechend ist eine konstruktive Zusammenarbeit über die beteiligten Fachdisziplinen wie auch Berufszweige hinweg unabdingbar für eine patientenzentrierte Betreuung. Die FMCH und die FMH unterstützen ihre Organisationen und Mitglieder in dieser Herausforderung. Wir danken allen, welche sich aktiv zusammen mit uns hierfür einsetzen!

Dr. med. Josef E. Brandenberg, Präsident FMCH, Dr. med. Christoph Bosshard, Vizepräsident FMH

\title{
Der perioperative Behandlungsprozess
}

\author{
Sandra Becker, Sina Grape, Christoph Ellenberger, Christof Heim, Sebastian Krayer, Alex Noser, \\ Christine Zehntnera
}

Kommission für Struktur- und Prozessfragen, Schweizerische Gesellschaft für Anästhesiologie und Reanimation (a Präsidentin). Im Auftrag und mit Empfehlung des Vorstands der SGAR

Die Literatur zu diesem Artikel finden Sie online unter www.saez.ch $\rightarrow$ aktu elle Ausgabe oder $\rightarrow$ Archiv $\rightarrow 2018 \rightarrow$ Ausgabe 42
Aus redaktionellen Gründen und zugunsten der Lesbarkeit wird im Text die männliche oder weibliche Form für beide Geschlechter verwendet.

Die deutsche Fassung ist

Stammversion

Die Zusammenarbeit im perioperativen Behandlungsprozess ist eine äusserst anspruchsvolle Aufgabe, da viele Fachbereiche und Berufsgruppen ihre Interessen wahren und ihre Aktivitäten in komplexen Organisationsstrukturen aufeinander abstimmen müssen. Die Erarbeitung und Umsetzung patientengerechter und praxistauglicher Behandlungspfade unter Berücksichtigung der gültigen Vorgaben muss die Aufgabe der einzelnen Spitäler und operativen Behandlungszentren sein. Die Fach- und Berufsvereine, H+, Qualitätsgremien, Kantone, Bund etc. sind aufgerufen, dazu - wo notwendig - Richtlinien vorzugeben.

\section{Problemstellung}

Ein qualitativ hochstehendes Gesundheitswesen mit ausgewiesener Effizienz und Effektivität erfordert im Behandlungsprozess eine enge und dynamische Kooperation verschiedener spezialisierter Berufsgruppen. Diese Zusammenarbeit kann jedoch aufgrund von z.T. widersprüchlichen Bedürfnissen der Beteiligten erschwert und zusätzlich infolge fehlender struktureller Voraussetzungen empfindlich gestört werden. Das komplexe Umfeld im OP ist typischerweise gekennzeichnet durch Unterschiede in Denkweisen, Funktionen und Erwartungen. Wenn zwischen den Beteiligten im Behandlungsprozess ein Ungleichgewicht besteht oder sich eine Profession gegenüber den anderen (zu) dominant durchsetzt und Unschärfen bei der Abgrenzung der Aufgaben und Zuständigkeiten bestehen, kann dies die Zusammenarbeit behindern und die Effizienz der Behandlung und auch die Arbeitszufriedenheit der Beteiligten spürbar beeinträchtigen.
Eine gegenseitige fachliche und persönliche Akzeptanz der beteiligten Akteure, eine flache Hierarchie, direkte Kommunikation und Erreichbarkeit sowie die Bereitschaft zu flexibel adaptiertem Handeln sind für eine erfolgreiche partizipative Zusammenarbeit nicht nur förderlich, sondern Voraussetzungen.

Die Planung und Durchführung von operativen Eingriffen ist ein Musterbeispiel für die vernetzte interdisziplinäre und interprofessionelle Zusammenarbeit im medizinischen Behandlungsprozess. Für ein reibungsloses Gelingen müssen im perioperativen Ablauf vom Erstkontakt bis zum Fallabschluss verschiedene Spezialisten koordiniert und eng miteinander kooperieren. Neben Operateur und Anästhesist sind im OPBetrieb weitere Berufsgruppen wie die Fachleute für Operationstechnik, Anästhesie- und Lagerungspflege als eingespieltes Team für den Erfolg eines Eingriffs ausschlaggebend. Hinzu kommen bedarfsweise unterstützende Dienste wie z.B. Radiologie, Labormedizin und Blutspende sowie ggf. weitere spezialisierte Thera- 
peuten (z.B. Physio- und Ergotherapie) und andere Fachpersonen.

Einzelne Aufgaben und Behandlungsschritte werden nach- oder nebeneinander durch die unterschiedlichen Akteure entweder eigenverantwortlich oder in Aufgabenteilung geplant und durchgeführt. Da kaum alle Beteiligten gleichzeitig über alle notwendigen Informationen zum Behandlungsprozess des Patienten verfügen, ist das zielorientierte Zusammenspiel der Berufsgruppen eine herausfordernde Aufgabe. Zur Gewährleistung des Informationsaustausches gehören - neben multilateral vereinbarten Behandlungskonzepten und -pfaden - regelmässige Rapporte, Fallbesprechungen, Intervisionen und Supervisionen.

Sowohl die "Erklärung Sichere Chirurgie» [1] der Stiftung Patientensicherheit Schweiz, die Charta «Zusammenarbeit der Fachleute im Gesundheitswesen» [2] der Schweizerischen Akademie der Medizinischen Wissenschaften wie auch die "Helsinki Declaration on Patient Safety in Anesthesiology" [3] formulieren Handlungsempfehlungen zur medizinischen Qualitätssicherung und -verbesserung. Eine optimale Koordination der Arbeiten verspricht ein hohes Synergiepotential mit gesteigerter Flusseffizienz und erhöhter Produktivität im Team.

Aufgrund der heterogenen Strukturen der unterschiedlichen Institutionen (Spitäler, Kliniken, operative Zentren, Praxen) und wegen z.T. divergierender Partikularinteressen der involvierten Berufsgruppen sowie unter Umständen unterschiedlicher fachspezifischer Vorgaben ist die Vorstellung, ein einfaches, allgemeingültiges und allseitig akzeptiertes Konzept für den perioperativen Behandlungsprozess festlegen $\mathrm{zu}$ können, wohl unrealistisch.

Hingegen sind die einzelnen operativ tätigen Institutionen aufgrund ihrer Organisationsverantwortung verpflichtet, die Zuständigkeiten zwischen ihren beteiligten Akteuren auf der lokalen Ebene zu regeln. Voraussetzung dazu sind griffige, den fachlichen Rahmen bildende Vorgaben, die - wo notwendig - primär im Konsens durch die operativ und invasiv tätigen Fachgesellschaften unter dem Dach der FMCH definiert werden müssen. Sie sind somit aufgefordert, die fachspezifische Umsetzung zu initiieren und mit den beteiligten Fach- und Berufsvereinen, den Dachverbänden und den nationalen Gesundheitsgremien zu koordinieren.

Im Folgenden werden exemplarische Beispiele von verschiedenen therapeutischen und interventionellen Massnahmen im Verlauf des perioperativen Prozesses aufgezeigt, wo sich die Notwendigkeit zu klaren Absprachen und Regelungen in den einzelnen Institutionen aufdrängt.

\section{Präoperative Phase - gezielte Evaluation mit Risiko-Assessment}

Der operierende und invasiv tätige Facharzt steht als Initiator und meist auch als Fallführer primär in der planerischen Verantwortung. Er führt i.d.R. nur einen Teil der Verordnungen und Aktionen selbst durch und verteilt resp. delegiert die weiteren nicht operativfachspezifischen Aufgaben. Sofern die Operation mit Anästhesiebeteiligung erfolgt, teilt er sich die Verantwortung in verschiedenen Teilbereichen mit dem Anästhesisten. Dabei müssen verschiedene Aspekte in Bezug auf den geplanten operativen Eingriff berücksichtigt und ggf. geklärt werden:

- Abgestimmte Planung der präoperativen Patiententermine und interdisziplinäre Koordination der Sprechstunden auch bezüglich Inhalten (Anamnese, körperliche Untersuchung, allgemeine Informationen zu Behandlungsablauf und OP-Vorbereitung etc.);

- Aufklärung und Einholen des Einverständnisses des Patienten (Informed Consent [4]), sowohl zum chirurgischen wie auch zum anästhesiologischen Vorgehen;

- Abklärungsstandards für spezifische Patientengruppen und Eingriffe;

- Zeitgerechte Initiierung spezifischer Abklärungen (Lungenfunktion, Echokardiographie etc.) und speziell bei Patienten mit relevanten Begleiterkrankungen und entsprechend erhöhtem Risiko die Behandlung/Therapieoptimierung von Begleiterkrankungen wie arterieller Hypertonie, COPD etc.;

- Patient Blood Management (Blutprodukte): Einschätzen des perioperativen Blutverlustes und entsprechende Aufklärung des Patienten im Falle eines relevanten Blutungsrisikos. Die zeitgerechte Indikation spezifischer Abklärungen [4] sowie eine präoperative Anämiebehandlung sollen ermöglicht werden [5];

- Für Patienten, welche Blutprodukte ablehnen (z.B. Zeugen Jehovas), müssen verbindliche Richtlinien vorliegen;

- Patientenwille: Festlegen, wie der geäusserte Wille (z.B. Patientenverfügung) perioperativ beachtet und umgesetzt wird.

Bei Bedarf ist auch der Einbezug anderer Berufsgruppen zu koordinieren:

- Physiotherapie: z.B. zur Prehabilitation oder zur präoperativen Gehschulung bzw. zur Einübung der Extremitäten-Entlastung an Krücken und anderen Gehhilfen;

- Intensivmedizin: Information des Patienten über eine gegebenenfalls postoperative Betreuung in der Intensivstation oder Intermediate Care Unit. 


\section{Intraoperative Phase - klar geregelte Zuständigkeiten}

Auch für die intraoperative Phase sind verschiedene Themengebiete auf institutioneller Ebene zu regeln:

\section{Verantwortlichkeit und Zuständigkeiten für:}

- Strahlenschutz für Patienten und Mitarbeiter im OP entsprechend den Vorgaben des BAG: Organisation von Dosimetrie, obligatorischer Weiter- und Fortbildung des strahlenexponierten Personals u.v.m. Wartung von Geräten und Strahlenschutzmitteln;

- Erstellen und Einhalten von Hygienerichtlinien im OP: Infektionsprävention (z.B. Händedesinfektion, Desinfektion des OP-Gebietes). Reinigung, Desinfektion und Entsorgung. Schutz- und Isolierungsmassnahmen bei übertragbaren Erregern;

- Personalschutz: Arbeitsplatzsicherheit, Vorgehen bei Stich-/Schnittverletzungen etc.

\section{Einsatz und Anwendung von Checklisten [1]}

Als zentrale Massnahme zur Risikominderung bei der Vorbereitung und Durchführung einer Behandlung hat sich die Anwendung von Checklisten etabliert. Definierte Richtlinien, Schulungen und ständige Repetition sind für deren korrekte Anwendung essenziell. Checklisten dienen so speziell zur Vermeidung von fahrlässiger Unachtsamkeit im Rahmen der klinischen Routine und auch zur medicolegalen Absicherung.

\section{Lagerung des Patienten während des Eingriffs} Der Operateur benötigt für den Eingriff in der Regel eine spezifische Lagerung des Patienten, bei der patientenspezifische Faktoren berücksichtigt und die Interessen verschiedener Fachgruppen miteinbezogen werden müssen. Lagerungsschäden (Druckschäden an Nerven und Gewebe, Ischämien von Extremitäten etc.) müssen vermieden werden.

- Für die eingriffsspezifische Patientenlagerung während der Operation sollen lokal vereinbarte Standards vorliegen;

- Die Zuständigkeiten für deren Durchführung sollen geklärt sein;

- Die Durchführung und die Kontrolle der Lagerung sind zu dokumentieren;

- Eine Aufklärung des Patienten über lagerungsspezifische Risiken und Komplikationen [6] soll rechtzeitig erfolgen und dokumentiert werden.

\section{Transfusionskonzept, Gerinnungs- management und Hämovigilanz}

Sofern an einer Institution Operationen mit einem relevanten Blutungsrisiko durchgeführt oder z.B. Patien- ten mit Störungen der Blutgerinnung behandelt werden, müssen folgende Aspekte beachtet werden:

- Es bedarf eines Konzepts, das die Tranfusionspraxis und das Gerinnungsmanagement gemäss gültigen Richtlinien beschreibt. Dies soll auch einen Blutungsalgorithmus [7] enthalten;

- Unter Beachtung einer strengen Indikationsstellung und unter Abwägung potentieller Risiken muss eine ausreichende Menge von Blut- und Gerinnungsprodukten unter Berücksichtigung der institutionellen logistischen Faktoren (z.B. Verfügbarkeit von maschineller Autotransfusion [Cellsaver], Blutbank, Lagervorrat und Transportlogistik labiler Blut- und Gerinnungsprodukte) sichergestellt werden;

- Die Zuständigkeit für den Zeitpunkt und die Art der Durchführung von Transfusionen [8] sowie die Sicherheitsaspekte [9] im Rahmen der Hämovigilanz [10] müssen geregelt sein.

\section{Vorbestehende und adjuvante Medikation: Dosierung, Indikation und Zeitpunkt \\ der Verabreichung}

Das perioperative Medikamentenmanagement von Patienten unter Dauermedikation soll entsprechend der jeweiligen Medikamentenklasse festgelegt werden. Insbesondere bei einer vorbestehenden Therapie mit Antikoagulanzien und Thrombozyten-Aggregationshemmern müssen die Zielwerte der Gerinnung zum Operationszeitpunkt abhängig vom eingriffsspezifischen Blutungsrisiko und vom patientenspezifischen Thromboembolierisiko festgelegt und abgesprochen werden. Es empfiehlt sich, das Vorgehen und die $\mathrm{Zu}$ ständigkeit für das perioperative Management (Weiterführen, Pausieren, Revertieren oder Bridgen) anhand klarer und verbindlicher Richtlinien zu regeln.

Weitere Themenfelder zu Fragen der perioperativen Medikation umfassen auch:

- Richtlinien für die perioperative medikamentöse Thromboembolie-Prophylaxe;

- Richtlinien für die zeitgerechte Verabreichung der präoperativen Antibiotikagabe [11] zur Infektionsprophylaxe und -therapie;

- Endokarditis-Prophylaxe bei spezifischem kardialem Risiko;

- Zeitgerechte Verabreichung parenteraler Medikamente (z.B. Antifibrinolytika, Steroide) entsprechend den eingriffs- und klinikspezifischen Behandlungspfaden.

\section{Blutsperre/Blutleere [12, 13]}

Die Anwendung einer Blutsperre/Blutleere ermöglicht bei vielen Extremitäteneingriffen optimale Operations- 
bedingungen mit geringem Blutverlust. Aufgrund der potentiellen Risiken von Tourniquets speziell bei vorbestehenden Durchblutungsstörungen sind mögliche Kontraindikationen oder Zeitlimitationen zu kommunizieren:

- Definition der Richtwerte von maximalem Manschettendruck und ununterbrochener Ischämiedauer;

- Vorgehen bei Vorliegen von venösen und/oder arteriellen Durchblutungsstörungen;

- Applikationsart und -zeitpunkt der perioperativen Antibiotikaprophylaxe in Bezug zu Start und Ende der Ischämiephase bei Indikation einer Blutsperre/ Blutleere.

\section{Blasenkatheter [14]}

Die perioperative Einlage eines Blasenkatheters kann aus unterschiedlichen Gründen notwendig sein. $\mathrm{Zu}$ klärende Punkte sind:

- Indikationen zur kurzzeitigen oder länger dauernden Blasenentleerung (oder -füllung) über einen transurethralen Blasenkatheter;

- Indikationen für eine suprapubische Blasenableitung (Zystofix);

- Vorgaben zur Hygiene und ggf. antibiotischer Prophylaxe oder Therapie bei liegendem Blasenkatheter, insb. bei Eingriffen mit Implantation von Fremdmaterial (Gelenkprothetik, Gefäss- und Herzklappeneingriffe).

\section{Wärmemanagement [15]}

Eine Hypothermie $\left\langle 35,5^{\circ} \mathrm{C}\right.$ kann auch perioperativ zu schwerwiegenden Komplikationen führen. Dazu zählen kardiale Ereignisse wie Herzrhythmusstörungen und -infarkte, Gerinnungsstörungen und Blutungsneigung mit erhöhtem Transfusionsbedarf, Wundinfektionen und Druckulzera. Damit beeinflusst eine akzidentelle Hypothermie die Qualität des postoperativen Resultats entscheidend mit.

- Das Behandlungsteam sollte über die Risiken einer Hypothermie bei chirurgischen Patienten geschult werden. Ein Augenmerk ist insb. auch auf die erst postoperativ auftretende Hypothermie zu legen;

- Verbindliche Richtlinien und Massnahmen zur Vermeidung einer Hypothermie sollen vorliegen und deren Wirkung periodisch überprüft werden.

\section{Postoperative Phase}

\section{Postoperative Schmerztherapie [16]}

Postoperative Wundschmerzen dürfen keine «übliche» Begleiterscheinung einer operativen Behandlung sein. Die Möglichkeiten einer adäquaten Schmerztherapie sind grundsätzlich vorhanden und können das post- operative Morbiditäts- und Mortalitätsrisiko reduzieren. Die postoperative Schmerztherapie stützt sich auf folgende Voraussetzungen:

- Regelmässige Schmerzerfassung und standardisierte Dokumentation der Werte und Massnahmen;

- Festlegung von Interventionsgrenzen und -massnahmen;

- Erstellung klinikeigener Behandlungsstandards (standard operating procedures, SOP) zur postoperativen Schmerztherapie, insbesondere prozedurenspezifische Analgesiekonzepte;

- Erstellung klinikeigener Behandlungsstandards für die Prophylaxe und Therapie von Nebenwirkungen der Analgetika.

\section{Postoperative Überwachung [17]}

Die Betreuung in der unmittelbar postoperativen Phase liegt in der gemeinsamen Verantwortung von Anästhesist und Operateur (bzw. des diagnostisch oder interventionell tätigen Arztes). Folgende Voraussetzungen müssen gegeben sein:

- Geeignete Infrastruktur zur postoperativen Nachbetreuung einschliesslich der Möglichkeit zur notfallmässigen Intervention bei kardiovaskulären, pulmonalen, neurologischen, gastroenterologischen u.a. Komplikationen;

- Die betreuenden Pflegepersonen sind spezifisch ausgebildet für das Erkennen und Behandeln eingeschränkter Vitalfunktionen und die selbständige überbrückende Behandlung bei unerwarteten Ereignissen;

- Jedes Spital resp. Operationszentrum soll geeignete Leitlinien und Standards für die postoperative Betreuung von Patienten formulieren. Dazu gehört auch die Festlegung von verbindlichen Entlassungskriterien nach ambulant und tageschirurgisch durchgeführten Eingriffen.

\section{Fazit}

Die Zusammenarbeit im operativen Behandlungsprozess ist eine sehr anspruchsvolle Herausforderung, da viele Fachbereiche ihre Interessen aufeinander abstimmen müssen.

Klar definierte und kommunizierte Prozessabläufe schaffen Transparenz.

Die Einsicht aller Beteiligten und deren Motivation und Wille zur konstruktiven Zusammenarbeit sind die besten Voraussetzungen für eine qualitativ hochstehende Behandlung unter optimalem Ressourceneinsatz. Positive Rückmeldungen und Zufriedenheit von Patienten und Beteiligten sollen so auch als Ansporn dienen für den weiteren kontinuierlichen Verbesserungsprozess. 